\title{
Method for Detecting Small Area Forgery by Comparing Two Document Images
}

\author{
Daejune $\mathrm{Ko}^{1}$ and ${ }^{*}$ Eui Chul Lee ${ }^{2}$ \\ ${ }^{1}$ Department of Computer Science, Graduate School, Sangmyung University \\ 20, Hongjimun 2-gil, Jongno-gu, Seoul 03016, KOREA \\ ${ }^{2}$ Department of Computer Science, Sangmyung University \\ 20, Hongjimun 2-gil, Jongno-gu, Seoul 03016, KOREA \\ ${ }^{1}$ kodaejune@gmail.com, 2 eclee@smu.ac.kr \\ *Corresponding author
}

\begin{abstract}
In this paper, a method for detecting tiny area with forgery between two images is proposed. The proposed method includes two procedures. Firstly, geometric features such as translation, rotate and scaling between two images are extracted by using SIFT algorithm. The false geometric correspondences are filtered out by cross validation. Then two images are aligned by affine transform using the best three corresponding pairs. Next, aligned images are divided locally and uniformly, the size of each region $20 \times 20$ pixels and solving slightly misaligned between two images is used by moving window method for determining the best aligned position. And local similarities between two images are measured by calculating correlation coefficient. To verify proposed method, ten receipts were used. Each receipt was saved as two types such as original and forgery ones, respectively. After training, we defined that the correlation coefficient as an optimal threshold was 0.9 for classifying non-forgery and forgery areas. At result, we acquired $7.391 \%$ equal error rate.
\end{abstract}

Keywords: forgery detection, image comparison, affine transform, geometric alignment, correlation coefficient

\section{Introduction}

By the rapid development of IT technology, digital data could be modified by anyone easily. Whereby, forgery of digital data has spread to various fields. In the early days of information technology, forgery of digital data had been used to hide individual identity of news image or augment reality in the field of computer graphics. However, in recent years, it has been used in criminal fields such as counterfeit banknotes or forgery documents.

In pervious works, several researches for forgery detection have been performed by using image or watermarking. Firstly, image based methods focused on copy-move forgery $[1,2,3]$. The main purpose of copy-move forgery is to hide or highlight specific area in the image. In these methods, forgeries were detected by analyzing local block features or keypoints. In local block-based method, images are subdivided for extracting features and every region matched another region [2]. In keypoint-based methods, features could be extracted and matched without subdivision [3]. Both, block- and keypoint-based methods include filtering feature process. However, these methods have strong dependencies with the quality of block feature of keypoints. Secondly, invisible watermarking based methods have been widely used [4,5]. Yoo et al. proposed a hybrid watermarking method [4]. They embed the PN-Sequence using wavelet transform and verified algorithm using various forgeries. Kim and Choi proposed quantization watermarking method. They used discrete cosine transform and adaptive quantization 
after discrete wavelet transform [5]. However, such methods can be defeated by compression or simple arithmetic operation of digital contents. Also, the mentioned methods have problem for detecting tiny (several pixels) forged region.

To solve these problems, a method for detecting tiny area with forgery between two images such as original and forgery ones is proposed. Geometric variation between two images are aligned by affine transform then tiny forged region is determined by using the optimal threshold of correlation coefficient. Detail of the proposed algorithm is explained in Section 2. In Section 3, experimental results using actual receipt images are given. Lastly, conclusion and future work plan are discussed.

\section{Proposed Method}

Our proposed method consists of two parts. First part is a process of aligning two images by considering geometric transformation such as translation, rotate and scaling between two images. Next, a process of detecting small area with forgery between two images is performed.

\subsection{Geometric Alignment with Cross Validation}

Two images of same document acquired by different time, machines and sensors can intend geometric variation as shown in Figure 1.
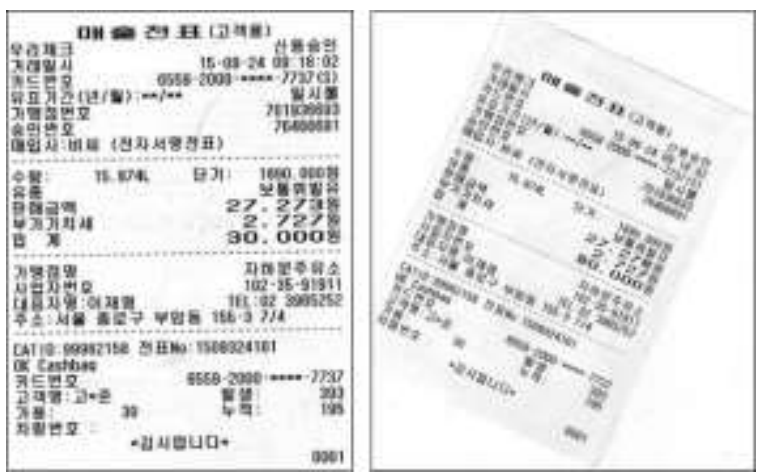

Figure 1. Example of Geometric Variation Between Two Images of Same Document

To compare two images for forgery detection, the images firstly should be aligned. The geometric alignment is performed as the flow of Figure 2.

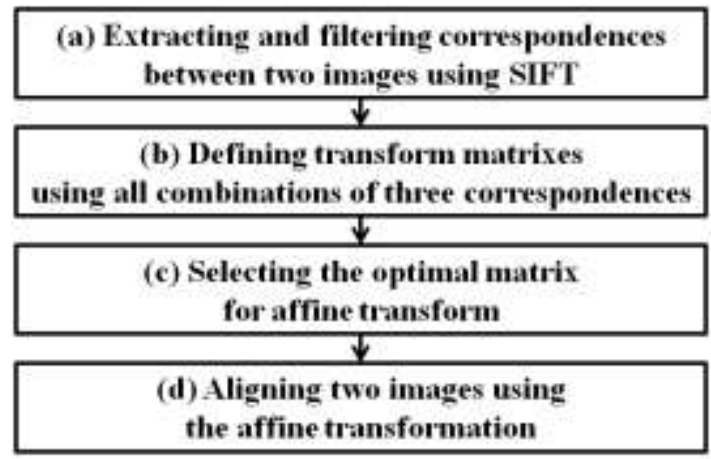

Figure 2. Processing Flow for Aligning Two Images

Corresponding pairs between two images are extracted by SIFT (Scale Invariant Features Transform) $[6,7]$ as Figure 2 (a). SIFT consists of two process. First process is 
to define feature vectors using key points as shown in Figure 3. In Figure 3, radius of circle means size of feature vector and direction of arrowed line means direction of feature vector.

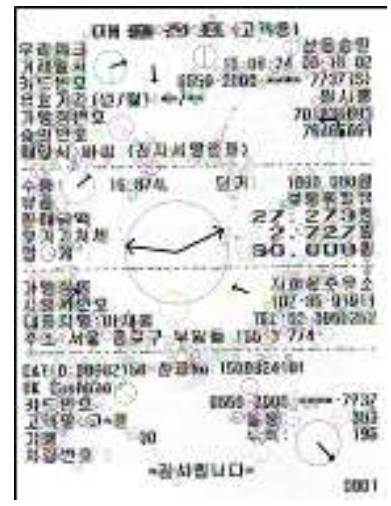

Figure 3. Key Points and Feature Vectors Extracted by SIFT

Next process is to match between feature vectors extracted from two images based on Euclidian distance. In other words, it was to find correspondences between two images as shown in Figure 4.

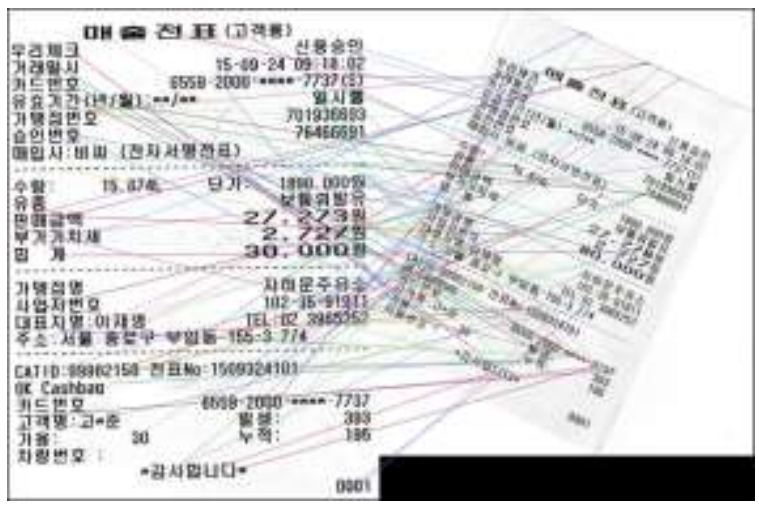

Figure 4. Initial Result of Defining Correspondences Using SIFT

As shown in Figure 4, because multiple positions of left image correspond to one position of right image, this phenomenon may occur ambiguity problem for defining geometric transform matrix. To remove such false corresponding pairs, our method adopts cross validation concept as shown in Figure 5. 


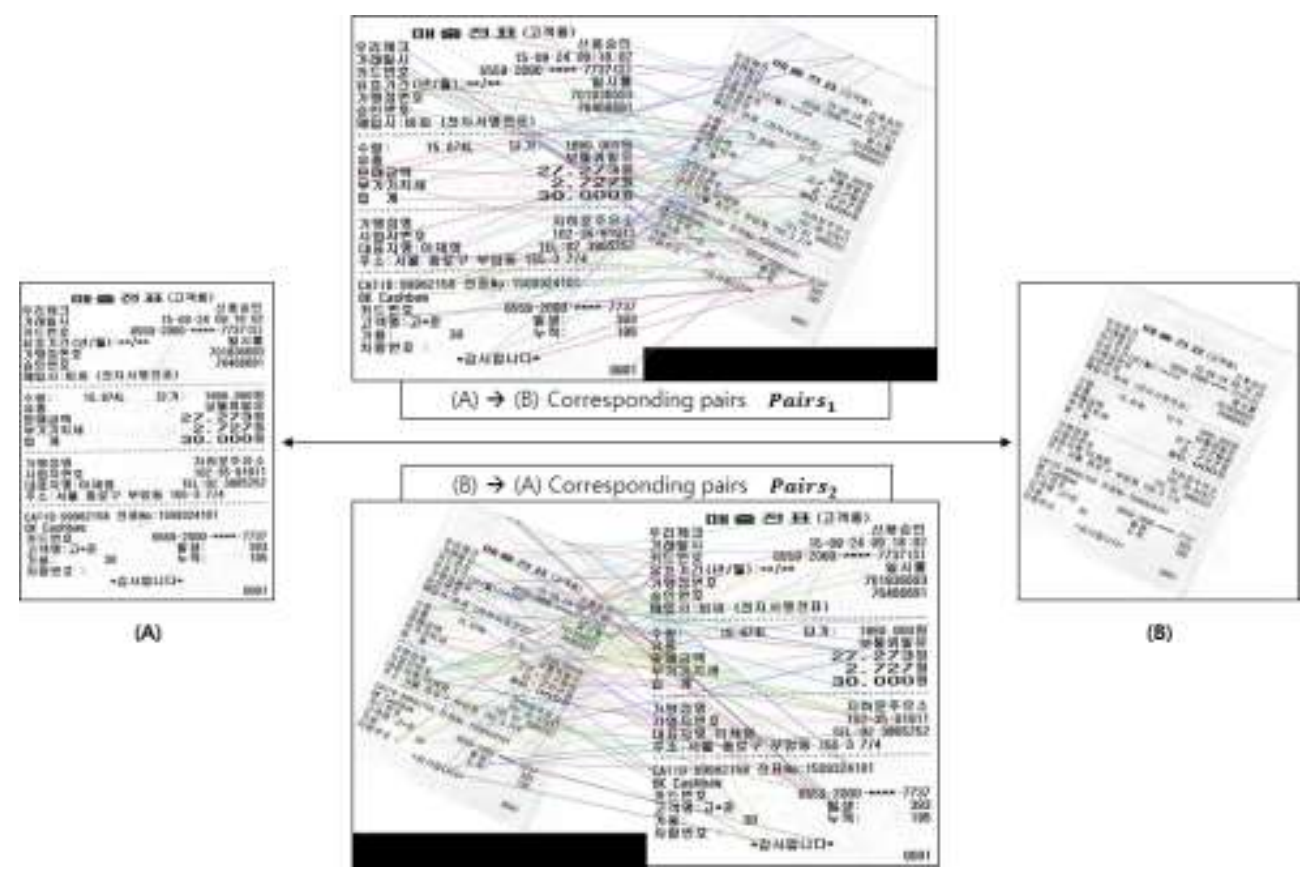

Figure 5. For Solving Same Targeted Points Process

In detail, Pairs 1 in Figure 5 is corresponding pairs from (A) to (B). Also, Pairs 2 is corresponding pairs from (B) to (A). Consequently, we selected the filtered correspondences with satisfying both Pairs 1 and Pairs 2 as shown in Figure 6.

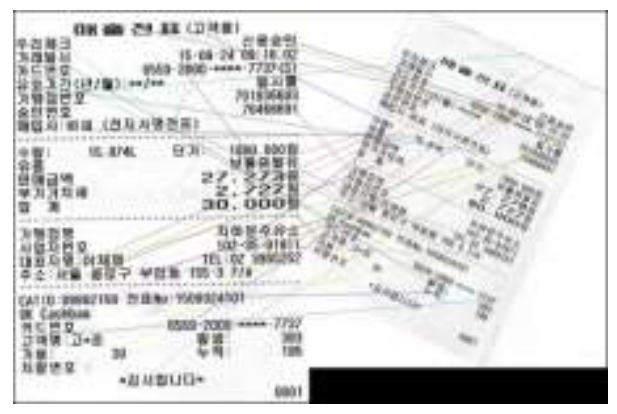

Figure 6. Filtered Correspondences with Satisfying Both Pairs ${ }_{1}$ and Pairs 2

In our proposed method, we assumed that geometric transform from scanner includes three factors such as scaling, rotation and translation as shown in Figure 7 [8].

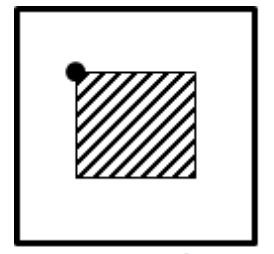

Original

$\left[\begin{array}{l}x \\ y \\ 1\end{array}\right]$

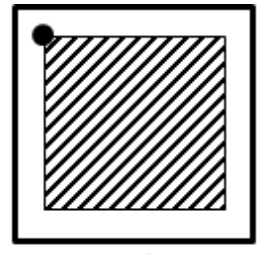

Scale

$\left[\begin{array}{ccc}S_{x} & 0 & 0 \\ 0 & S_{y} & 0 \\ 0 & 0 & 1\end{array}\right]$

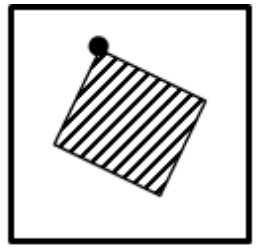

Rotate

$\left[\begin{array}{ccc}\cos \theta & -\sin \theta & 0 \\ \sin \theta & \cos \theta & 0 \\ 0 & 0 & 1\end{array}\right]$

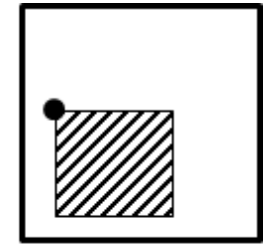

Translate

$\left[\begin{array}{ccc}1 & 0 & T_{x} \\ 0 & 1 & T_{y} \\ 0 & 0 & 1\end{array}\right]$

Figure 7. Geometric Transform Factor form Scanner 
As Figure 2 (b), transform matrixes are defined by using all combinations of three correspondences and equation (1).

$$
\left[\begin{array}{ccc}
x 1_{s} & x 2_{s} & x 3_{s} \\
y 1_{s} & y 2_{s} & y 3_{s} \\
1 & 1 & 1
\end{array}\right]\left[\begin{array}{ccc}
x 1_{s}^{\prime} & x 2_{s}^{\prime} & x 3_{s}^{\prime} \\
y 1_{s}^{\prime} & y 2_{s}^{\prime} & y 3_{s}^{\prime} \\
1 & 1 & 1
\end{array}\right]^{-1}=\left[\begin{array}{ccc}
S_{x} & 0 & 0 \\
0 & S_{y} & 0 \\
0 & 0 & 1
\end{array}\right]\left[\begin{array}{ccc}
\cos \theta & -\sin \theta & 0 \\
\sin \theta & \cos \theta & 0 \\
0 & 0 & 1
\end{array}\right]\left[\begin{array}{ccc}
1 & 0 & T_{x} \\
0 & 1 & T_{y} \\
0 & 0 & 1
\end{array}\right]
$$

In equation (1), $A^{-1}$ means inverse matrix $A .\left(x 1_{\mathrm{s}}, y 1_{\mathrm{s}}\right),\left(x 2_{\mathrm{s}}, y 2_{\mathrm{s}}\right),\left(x 3_{\mathrm{s}}, y 3_{\mathrm{s}}\right)$ are three

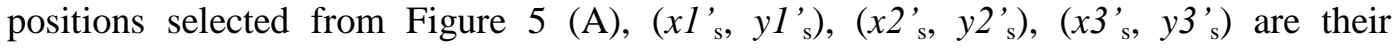
correspondences onto Figure 5 (B). Consequently, " $\left(x l_{\mathrm{s}}, y 1_{\mathrm{s}}\right)$ and $\left(x l_{\mathrm{s}}\right.$,,$\left.y l_{\mathrm{s}}\right)$ "), " $\left(x 2_{\mathrm{s}}, y 2_{\mathrm{s}}\right)$ and $\left(x 2_{\mathrm{s}}^{\prime}, y 2_{\mathrm{s}}^{\prime}\right)$ ", and " $\left(x 3_{\mathrm{s}}, y 3_{\mathrm{s}}\right)$ and $\left(x 3_{\mathrm{s}}^{\prime}, y 3_{\mathrm{s}}{ }_{\mathrm{s}}\right.$ " " are the corresponding pairs for determining affine transform matrix to align.

Next, as Figure 2 (c) one matrix for affine transform is selected from transform matrixes by using equation (2) (3).

$$
\begin{aligned}
& \underset{[S T, \theta)}{\operatorname{argmin}}(|a-e|+|b+d|) \\
& {\left[\begin{array}{ccc}
S \cdot \cos \theta & -S \cdot \sin \theta & T_{x} \cdot S \cdot \cos \theta-T_{y} \cdot S \cdot \sin \theta \\
S \cdot \sin \theta & S \cdot \cos \theta & T_{x} \cdot S \cdot \sin \theta+T_{y} \cdot S \cdot \cos \theta \\
0 & 0 & 1
\end{array}\right]=\left[\begin{array}{ccc}
a & b & c \\
d & e & f \\
0 & 0 & 1
\end{array}\right]}
\end{aligned}
$$

In equation (2), if the one among transform matrices is equivalent with the genuine affine transform matrix, $a+e$ and $b-d$ should be 0 . Because $S_{x}$ and $S_{y}$ are scale factor in geometric transform and have same value $(S)$ in scanner, right side of the equation (1) is equal to left side of equation (3). Lastly, two images are aligned by using equation (4).

$$
\left[\begin{array}{l}
x_{s} \\
y_{s} \\
1
\end{array}\right]=\left[\begin{array}{lll}
a & b & c \\
d & e & f \\
0 & 0 & 1
\end{array}\right]\left[\begin{array}{c}
x_{s}^{s} \\
y_{s}^{b} \\
1
\end{array}\right]
$$

In equation (4), $W$ and $H$ are width and height of Figure 5 (A) image, respectively. And $\left(x_{\mathrm{s}}, y_{\mathrm{s}}\right)$ and $\left(x_{\mathrm{s}}^{\prime}, y_{\mathrm{s}}^{\prime}\right)$ are the correspondence of two comparing images such as Figure 5 (A) and (B). As a result, non-aligned image as Figure 8 (b) is geometrically transformed as aligned one as shown in Figure 8 (c).

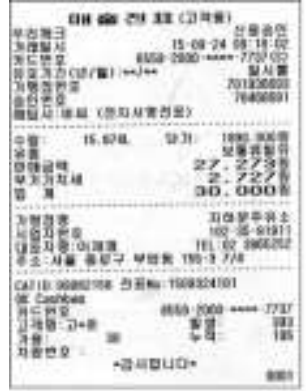

(a)

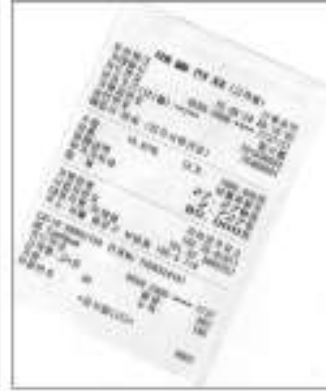

(b)

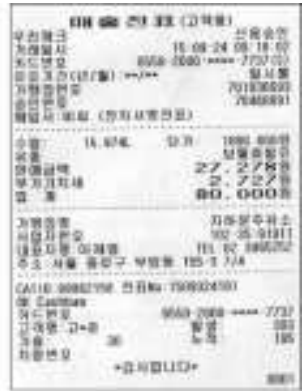

(c)

Figure 8. Result of Alignment by Affine Transform (a) Target Image, (b) Nonaligned Image, (c) Aligned Image of (b)

\subsection{Calculating Correlation Coefficient Small Forgery Area between Two Images}

For detecting small forgery area between two images, each image is divided by $20 \times 20$ pixels as shown in Figure 9. 


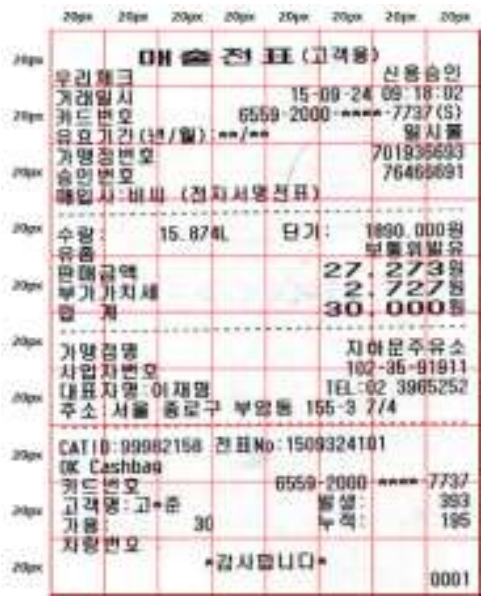

Figure 9. Example of Divided Regions by $20 \times 20$ Pixels

For solving slightly misaligned image, the highest correlation coefficient of corresponding small areas between two images by using moving window method were selected as shown in Figure 10 [9].

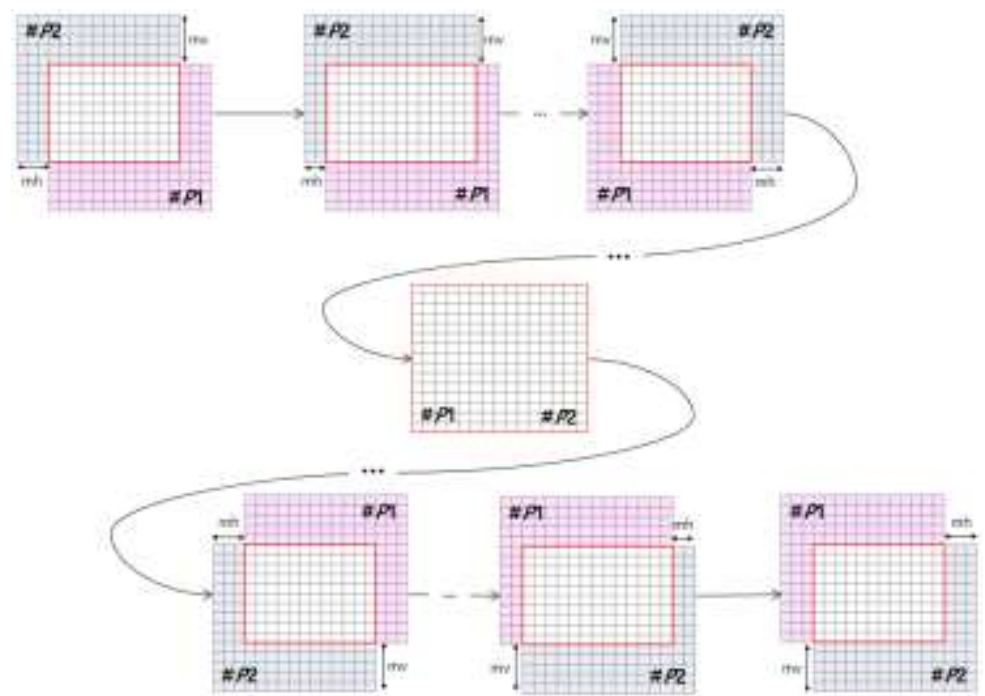

Figure 10. Moving Window Method [9]

Correlation coefficient of corresponding small areas between two images were calculated by equation (4) [10].

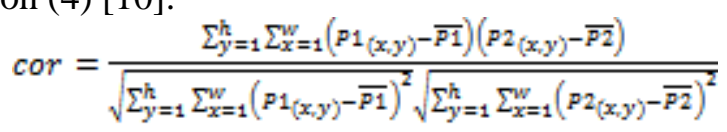

In equation (4), $P 1$ is a small area in original image, $P 2$ is a small area in aligned image. And $P 1$ and $P 2$ are corresponding areas to each other. $P 1_{(x, y)}$ and $P 2_{(x, y)}$ mean a pixel intensity of the small areas on two images, respectively. $(x, y)$ means pixel position of small area, $h$ and $w$ mean height and width of small area, respectively. In our method, both $w$ and $h$ are configured as 18 20. $\overline{P 1}$ and $\overline{P 2}$ are the averages intensity of each region which can be calculated by equation (5).

$$
\bar{P}=\frac{\sum_{y=1}^{\mathrm{h}} \Sigma_{x=1}^{W} P_{(x, y)}}{w \times h}
$$




\section{Experiment Result}

Real five receipts were used for this experiment. Firstly, the receipts were scanned without forgeries and cropped without background. Next, we manually made more than five forgeries per one receipt image such as modifying total money, card number and date as shown in Figure 11.

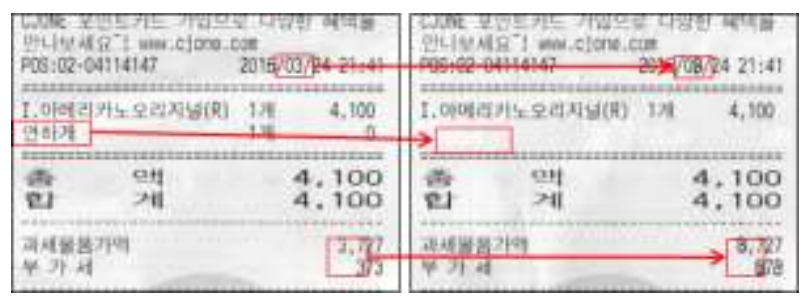

Figure 11. Example of Forged and Non-Forged Receipt Pair

Then, the modified receipt was scanned into more than five images with variations such as angle, dots per inch, and position. The variations mean affine factors such as rotation, scaling, and translation, respectively. All scanned images for the experiment were changed to 8-bit gray scale ones. Information of experimental images are shown in Table 1.

Table 1. Information of Experimental Receipts

\begin{tabular}{|c|c|c|c|c|c|}
\hline & Receipt \#1 & Receipt \#2 & Receipt \#3 & Receipt \#4 & Receipt \#5 \\
\hline Images & 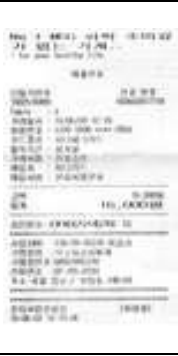 & 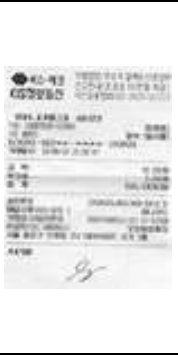 & 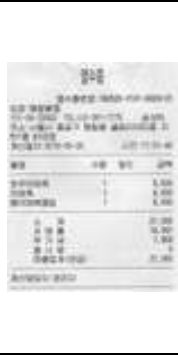 & 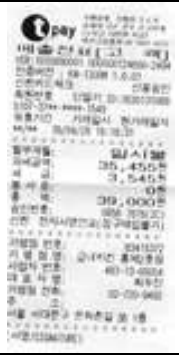 & 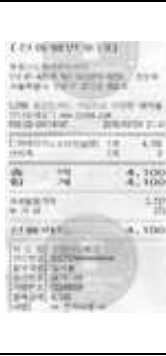 \\
\hline $\begin{array}{c}\text { Number of } \\
\text { Forgeries }\end{array}$ & 6 & 7 & 5 & 7 & 7 \\
\hline $\begin{array}{l}\text { Number of } \\
\text { geometric } \\
\text { variations }\end{array}$ & 6 & 8 & 6 & 6 & 6 \\
\hline $\begin{array}{l}\text { Image } \\
\text { Size }\end{array}$ & $500 \times 860$ & $580 \times 760$ & $580 \times 780$ & $400 \times 860$ & $560 \times 920$ \\
\hline $\begin{array}{c}\text { Number of } \\
\text { local area } \\
\text { with forgery }\end{array}$ & 8 & 7 & 28 & 24 & 26 \\
\hline
\end{tabular}

\begin{tabular}{|c|c|c|c|c|c|}
\hline & Receipt \#6 & Receipt \#7 & Receipt \#8 & Receipt \#9 & Receipt \#10 \\
\hline Images & 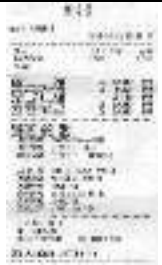 & 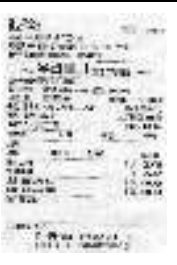 & 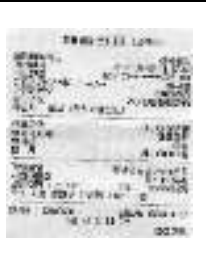 & 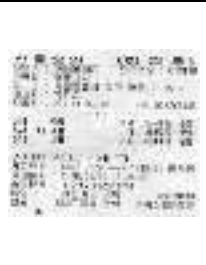 & 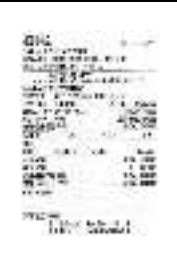 \\
\hline $\begin{array}{c}\text { Number of } \\
\text { Forgeries }\end{array}$ & 5 & 8 & 6 & 5 & 10 \\
\hline $\begin{array}{l}\text { Number of } \\
\text { geometric } \\
\text { variations }\end{array}$ & 5 & 6 & 7 & 9 & 7 \\
\hline
\end{tabular}




\begin{tabular}{|c|c|c|c|c|c|}
\hline $\begin{array}{c}\text { Image } \\
\text { Size }\end{array}$ & $527 \times 925$ & $905 \times 1325$ & $547 \times 615$ & $573 \times 497$ & $882 \times 1310$ \\
\hline $\begin{array}{c}\text { Number of } \\
\text { local area } \\
\text { with forgery }\end{array}$ & 19 & 27 & 17 & 14 & 39 \\
\hline
\end{tabular}

To define the threshold for classifying between forgery and non-forgery areas, correlation coefficients were firstly collected for all comparison cases. As shown in table 2 , approximately $95 \%$ of non-forgery areas had correlation coefficient of at least 0.8 (bold in Table 2). In cases of receipt \#9, approximately 5\% of non-forgery areas had correlation coefficient less than 0.8 (underlined values in Table 2).

Table 2. Correlation Coefficient of Non-Forgery Area

\begin{tabular}{|c|c|c|c|c|c|}
\hline & Receipt \#1 & Receipt \#2 & Receipt \#3 & Receipt \#4 & Receipt \#5 \\
\hline $0.0 \leq$ cor $<0.1$ & $0.000 \%$ & $0.000 \%$ & $0.000 \%$ & $0.000 \%$ & $0.000 \%$ \\
\hline $0.1 \leq$ cor $<0.2$ & $0.000 \%$ & $0.000 \%$ & $0.000 \%$ & $0.000 \%$ & $0.000 \%$ \\
\hline $0.2 \leq \operatorname{cor}<0.3$ & $0.000 \%$ & $0.000 \%$ & $0.000 \%$ & $0.000 \%$ & $0.000 \%$ \\
\hline $0.3 \leq$ cor $<0.4$ & $0.000 \%$ & $0.000 \%$ & $0.000 \%$ & $0.000 \%$ & $0.000 \%$ \\
\hline $0.4 \leq$ cor $<0.5$ & $0.000 \%$ & $0.000 \%$ & $0.000 \%$ & $0.000 \%$ & $0.013 \%$ \\
\hline $0.5 \leq \operatorname{cor}<0.6$ & $0.000 \%$ & $0.000 \%$ & $0.000 \%$ & $0.000 \%$ & $0.013 \%$ \\
\hline $0.6 \leq \operatorname{cor}<0.7$ & $0.000 \%$ & $0.137 \%$ & $0.000 \%$ & $0.020 \%$ & $0.185 \%$ \\
\hline $0.7 \leq$ cor $<0.8$ & $0.141 \%$ & $1.105 \%$ & $0.362 \%$ & $0.498 \%$ & $0.805 \%$ \\
\hline $0.8 \leq$ cor $<0.9$ & $2.421 \%$ & $4.545 \%$ & $1.055 \%$ & $4.679 \%$ & $4.261 \%$ \\
\hline \multirow[t]{2}{*}{$0.9 \leq \operatorname{cor}<1.0$} & $97.438 \%$ & $94.213 \%$ & $98.583 \%$ & $94.803 \%$ & $94.724 \%$ \\
\hline & Receipt \#6 & Receipt \#7 & Receipt \#8 & Receipt \#9 & Receipt \#10 \\
\hline $0.0 \leq \operatorname{cor}<0.1$ & $0.000 \%$ & $0.000 \%$ & $0.000 \%$ & $0.000 \%$ & $0.000 \%$ \\
\hline $0.1 \leq \operatorname{cor}<0.2$ & $0.000 \%$ & $0.000 \%$ & $0.000 \%$ & $0.000 \%$ & $0.000 \%$ \\
\hline $0.2 \leq$ cor $<0.3$ & $0.000 \%$ & $0.000 \%$ & $0.000 \%$ & $0.000 \%$ & $0.000 \%$ \\
\hline $0.3 \leq \operatorname{cor}<0.4$ & $0.000 \%$ & $0.000 \%$ & $0.000 \%$ & $\underline{0.000 \%}$ & $0.000 \%$ \\
\hline $0.4 \leq$ cor $<0.5$ & $0.000 \%$ & $0.006 \%$ & $0.000 \%$ & $\underline{0.000 \%}$ & $0.005 \%$ \\
\hline $0.5 \leq \operatorname{cor}<0.6$ & $0.000 \%$ & $0.085 \%$ & $0.018 \%$ & $\underline{0.155 \%}$ & $0.086 \%$ \\
\hline $0.6 \leq \operatorname{cor}<0.7$ & $0.034 \%$ & $1.081 \%$ & $0.252 \%$ & $1.770 \%$ & $1.071 \%$ \\
\hline $0.7 \leq$ cor $<0.8$ & $0.357 \%$ & $3.163 \%$ & $0.144 \%$ & $3.574 \%$ & $2.685 \%$ \\
\hline $0.8 \leq$ cor $<0.9$ & $3.127 \%$ & $10.238 \%$ & $1.621 \%$ & $5.464 \%$ & $10.320 \%$ \\
\hline $0.9 \leq \operatorname{cor}<1.0$ & $96.483 \%$ & $85.427 \%$ & $97.964 \%$ & $89.038 \%$ & $85.832 \%$ \\
\hline
\end{tabular}

Next, Table 3 shows the distributions of correlation coefficients in cases of comparison with forgery area. Approximately $90 \%$ of forgery areas excepting receipt \#5 had correlation coefficient of less than 0.9 (bold in Table 3 ). In case of receipt \#2, approximately $20 \%$ of forgery areas had correlation coefficient more than 0.9 (under line in Table 3).

Table 3. Correlation Coefficient of Forgery Area

\begin{tabular}{|c|c|c|c|c|c|}
\hline & Receipt \#1 & Receipt \#2 & Receipt \#3 & Receipt \#4 & Receipt \#5 \\
\hline $0.0 \leq$ cor $<0.1$ & $\mathbf{0 . 0 0 0 \%}$ & $0.000 \%$ & $\mathbf{0 . 0 0 0 \%}$ & $\mathbf{0 . 0 0 0 \%}$ & $\mathbf{0 . 0 0 0 \%}$ \\
\hline $0.1 \leq$ cor $<0.2$ & $\mathbf{0 . 0 0 0 \%}$ & $0.000 \%$ & $\mathbf{0 . 0 0 0 \%}$ & $\mathbf{0 . 0 0 0 \%}$ & $\mathbf{0 . 0 0 0 \%}$ \\
\hline $0.2 \leq$ cor $<0.3$ & $\mathbf{0 . 0 0 0 \%}$ & $0.000 \%$ & $\mathbf{0 . 0 0 0 \%}$ & $\mathbf{0 . 0 0 0 \%}$ & $\mathbf{0 . 0 0 0 \%}$ \\
\hline $0.3 \leq$ cor $<0.4$ & $\mathbf{0 . 0 0 0 \%}$ & $0.000 \%$ & $\mathbf{0 . 0 0 0 \%}$ & $\mathbf{0 . 0 0 0 \%}$ & $\mathbf{0 . 0 0 0 \%}$ \\
\hline $0.4 \leq$ cor $<0.5$ & $\mathbf{0 . 0 0 0 \%}$ & $0.000 \%$ & $\mathbf{0 . 0 0 0 \%}$ & $\mathbf{6 . 2 5 0 \%}$ & $\mathbf{0 . 0 0 0 \%}$ \\
\hline $0.5 \leq$ cor $<0.6$ & $\mathbf{0 . 0 0 0 \%}$ & $0.000 \%$ & $\mathbf{3 . 5 5 0 \%}$ & $\mathbf{6 . 9 4 4 \%}$ & $\mathbf{0 . 0 0 0 \%}$ \\
\hline $0.6 \leq$ cor $<0.7$ & $\mathbf{4 . 1 6 7 \%}$ & $0.000 \%$ & $\mathbf{2 4 . 8 5 2 \%}$ & $\mathbf{4 . 1 6 7 \%}$ & $\mathbf{3 . 8 4 6 \%}$ \\
\hline $0.7 \leq$ cor $<0.8$ & $\mathbf{6 6 . 6 6 7 \%}$ & $25.000 \%$ & $\mathbf{3 7 . 8 7 0 \%}$ & $\mathbf{5 1 . 3 8 9 \%}$ & $\mathbf{6 0 . 8 9 7 \%}$ \\
\hline
\end{tabular}




\begin{tabular}{|c|c|c|c|c|c|}
\hline $0.8 \leq$ cor $<0.9$ & $27.083 \%$ & $53.571 \%$ & $31.361 \%$ & $24.306 \%$ & $31.410 \%$ \\
\hline $0.9 \leq$ cor $<1.0$ & $2.083 \%$ & $21.429 \%$ & $2.367 \%$ & $6.944 \%$ & $3.846 \%$ \\
\hline & Receipt \#6 & Receipt \#7 & Receipt \#8 & Receipt \#9 & Receipt \#10 \\
\hline $0.0 \leq$ cor $<0.1$ & $0.000 \%$ & $0.000 \%$ & $0.000 \%$ & $0.000 \%$ & $0.000 \%$ \\
\hline $0.1 \leq \operatorname{cor}<0.2$ & $0.000 \%$ & $0.000 \%$ & $0.000 \%$ & $0.000 \%$ & $0.000 \%$ \\
\hline $0.2 \leq \operatorname{cor}<0.3$ & $0.000 \%$ & $0.000 \%$ & $0.000 \%$ & $0.000 \%$ & $1.099 \%$ \\
\hline $0.3 \leq$ cor $<0.4$ & $0.000 \%$ & $0.000 \%$ & $0.000 \%$ & $0.000 \%$ & $0.366 \%$ \\
\hline $0.4 \leq \operatorname{cor}<0.5$ & $0.000 \%$ & $3.704 \%$ & $0.000 \%$ & $0.000 \%$ & $4.029 \%$ \\
\hline $0.5 \leq$ cor $<0.6$ & $0.000 \%$ & $11.111 \%$ & $1.681 \%$ & $0.000 \%$ & $27.839 \%$ \\
\hline $0.6 \leq \operatorname{cor}<0.7$ & $5.263 \%$ & $17.901 \%$ & $33.613 \%$ & $23.016 \%$ & $20.879 \%$ \\
\hline $0.7 \leq$ cor $<0.8$ & $41.053 \%$ & $31.481 \%$ & $21.849 \%$ & $45.238 \%$ & $28.938 \%$ \\
\hline $0.8 \leq$ cor $<0.9$ & $44.211 \%$ & $29.012 \%$ & $34.454 \%$ & $25.397 \%$ & $12.821 \%$ \\
\hline $0.9 \leq$ cor $<1.0$ & $9.474 \%$ & $6.790 \%$ & $8.403 \%$ & $6.349 \%$ & $4.029 \%$ \\
\hline
\end{tabular}

As shown in Figure 12, correlation coefficients of forged area in new method show lower than previous method [11].
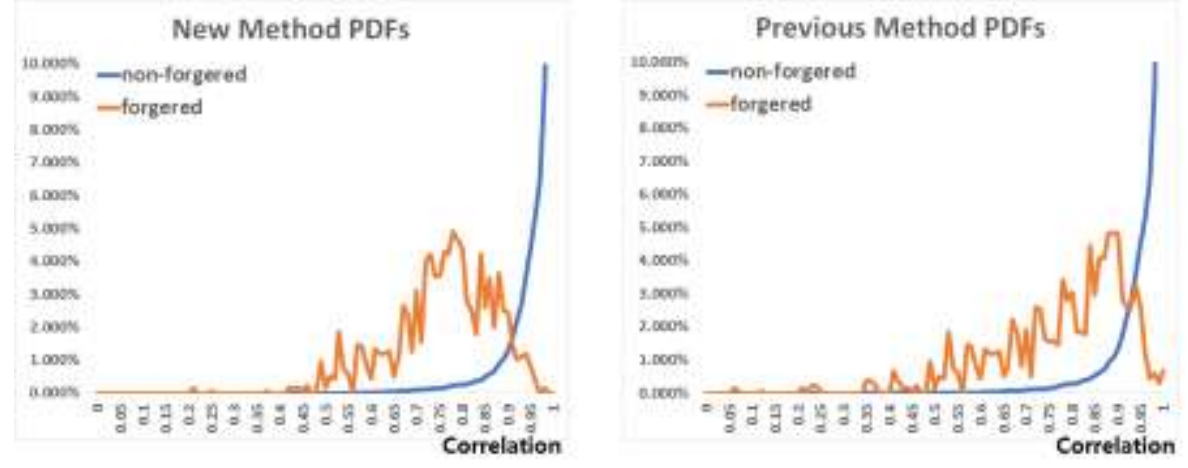

Figure 12. Example of Forged and Non-Forged Receipt Pair

As shown in Figure 13, EER (Equal Error Rate) of new method had $7.391 \%$ and EER of previous method had $12.639 \%$. Consequently, new method was improved about $5 \%$ than the previous method.

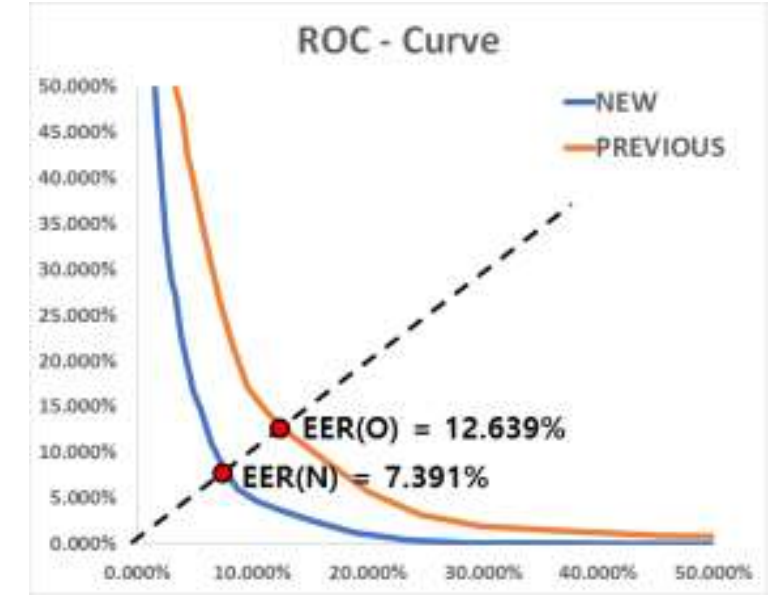

Figure 13. Example of Forged and Non-Forged Receipt Pair 


\section{Conclusions}

In this paper, small area forgery detection method was purposed. Firstly, geometric features between two images are extracted by using SIFT algorithm and filtered by comparing cross checking. Then two images are aligned by affine transform using the best three corresponding pairs. Next, aligned images are divided locally and uniformly, and solving slightly misaligned between two images is used by moving window method. As a result, almost non-forgery areas had correlation coefficient of more than 0.8 , almost forgery areas had correlation coefficient of less than 0.9 and EER had $7.391 \%$.

In the future works, sub-regions will be non-uniformly defined and detection method will be applied other method. Also image acquired from variety environments such as multiple scanners and smart phone camera will be tested.

\section{Acknowledgments}

This research was supported by the Bio \& Medical Technology Development Program of the NRF funded by the Korean government, MSIP(2016M3A9E1915855).

\section{References}

[1] V. Christlein, C. Riess, J. Jordan, C. Riess, and E. Angelopoulou, "An Evaluation of Popular CopyMove Forgery Detection Approaches", Journal of IEEE Transactions On Information Forensics And Security., vol. 7, no. 6, (2012), pp. 1841-1853

[2] B. Mahdian and S. Saic, "Detection of Copy-Move Forgery Using a Method Based on Blur Moment Invariants", Journal of Forensic Science International., vol. 171, no. 2, (2007), pp. 180-189

[3] J. Wang, G. Liu, Z. Zhang, Y. Dai, and Z. Wang, "Fast and Robust Forensics for Image RegionDuplication forgery", Journal of Acta Automatica Sinica, vol. 35, no. 12, (2009), pp. 1488-1495

[4] K. Yoo, G. Song, H. Choi, and W. Lee, "A Hybrid Digital Watermarking Technique for Copyright Protection and Tamper Detection on Still images", Journal of Review of Korean Society for Internet Information, vol. 4, no. 6, (2012), pp. 1841-1853

[5] J. Kim, H. Choi, "Adaptive Quantization Watermarking for Image Tamper-proofing", Proceedings of Korean Institute of Information Scientists and Engineers Conference, Gangwon, Korea, (2005) July 6-8

[6] D. Lowe, "Object Recognition from Local Scale-Invariant Features", proceedings of the seventh IEEE international conference, Kerkyra, Greece, (1999) September 20-27

[7] D. Lowe, "Distinctive Image Features from Scale-Invariant Keypoints", Journal of Computer Vision., vol. 60 , no. 2, (2004), pp. $91-110$

[8] R. Gonzalez, and R. Woods, "Digital Image Processing", PEARSON, (2009), pp. 109-111

[9] Y. Kim, H. Kim and E. C. Lee, "Emotion Classification Using Facial Temporal Sparsity", Journal of Applied Engineering Research., vol. 9, no. 24, (2014), pp. 24793-24801

[10] H. Han, "Method for Detecting Tiny Forged Areas between Two Images," HANBIT Media, (2009), pp. $72-80$.

[11] D. Ko, and E. C. Lee, "Method for Detecting Tiny Forged Areas between Two Images," Proceedings of Applied Science and Engineering for Better Human Life, Jeju, Korea, (2016).

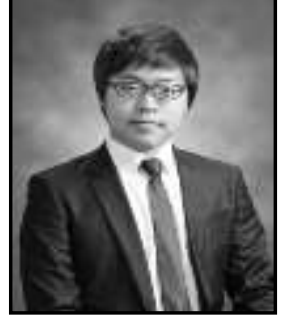

Daejune Ko, received a BS and MS degrees in the Department of Computer Science from Sangmyung University, Seoul, South Korea in 2013 and 2015, respectively. Currently, he has been Ph.D. course student in the Department of Computer Science from Sangmyung University since March 2015. His research interests include Pattern Recognition, Forgery Detection, OCR, 3D Computer Vision for Natural User Interface, and Biosignal Analysis. 


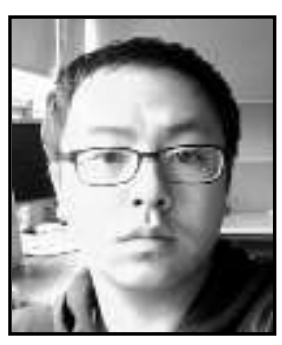

Eui Chul Lee, received a BS degree in Software from Sangmyung University, Seoul, South Korea in 2005. He received MS and Ph.D. degrees in Computer Science from Sangmyung University in 2007 and 2010, respectively. He was Researcher in Division of Fusion and Convergence of Mathematical Sciences at the National Institute for Mathematical Sciences from March 2010 to February 2012. Since March 2012, he has been Assistant Professor in the Department of Computer Science from Sangmyung University, Seoul, South Korea. His research interests include Computer Vision, Image Processing, Pattern Recognition, Forensic Science, and Affective Computing. 
International Journal of Signal Processing, Image Processing and Pattern Recognition Vol. 10, No. 6 (2017) 